\title{
GÉNERO Y DINERO: MÚLTIPLES MATICES EN LA RELACIÓN DE PAREJA
}

\author{
Vera Regina Xavier ${ }^{1}$ \\ Asociación Intercultural de Apoyo Emocional a la Diversidad Social, Bilbao, España
}

\begin{abstract}
RESUMEN. Este artículo es un estudio de caso de una pareja en proceso de petición de separación. Presentamos algunos de los conceptos culturales, sociales relacionados con el matrimonio. Estudiamos la relación a través de la interacción, del dinero, del trabajo y también de la desigualdad. Las sociedades estructuran y construyen su cultura en torno a la diferencia sexual de los individuos que la conforman, atribuyéndoles características y significados a las acciones que desempeñan mujeres y hombres o se espera que desempeñen, según lo socialmente aprendido. En la metodología presentamos un estudio de caso investigado en profundidad a través de una intervención de cinco encuentros. Presentando una discusión individual del caso interpretación general del tema y conclusión. El motivo fundamental de la investigación es aportar un otro enfoque de la relación, un dialogo provocativo para parejas y terapeutas.
\end{abstract}

Palabras clave: Género; poder; separación; conyugal.

\section{GÊNERO E DINHEIRO: OS MÚLTIPLOS MATIZES NA RELAÇAO DO CASAL}

RESUMO. Este artigo é um estudo de caso de um casal em processo de separação. Apresentamos alguns dos conceitos culturais e sociais relacionados ao casamento. Estudamos a relação através da interação entre trabalho, dinheiro e desigualdade. As sociedades estruturam e constroem sua cultura em torno da diferença sexual dos indivíduos que a compõem, atribuindo características e significados às ações que homens e mulheres desempenham, ou que se espera que desempenhem, conforme o socialmente aprendido. A metodologia utilizada é um estudo de caso desenvolvido em profundidade através de uma intervenção de cinco encontros em que se faz uma discussão individual do caso com interpretação geral do tema e conclusão. A principal razão para realizar a pesquisa é apresentar um enfoque diferente da relação, um diálogo provocativo para casais e terapeutas.

Palavras-chave: Gênero; poder; separação; conjugal.

\section{GENDER AND MONEY: THE MULTIPLE SHADES ON A COUPLE RELATIONSHIP}

ABSTRACT. This article is a case study of a couple in separation process request. We present some of the cultural concepts, social relating to marriage. We study the relationship through interaction, money, work and inequality. Structured societies and build your culture around sexual difference of the individuals comprising it, attributing characteristics and meanings to actions that men and women play or are expected to play, as socially learned. The methodology we present a case study investigated in depth through an intervention of five games. Presenting an individual case discussion general interpretation of the theme and conclusion.The main reason for the research is to provide a different approach to the relationship, a provocative dialogue for couples and therapists.

Keywords: Gender; power; separation; marital.

El objetivo general de este estudio de caso es conocer la doble perspectiva de los litigantes durante el período previo a la separación desde la interpretación de la estructura del texto obtenido a través de la transcripción de las entrevistas, enfocando los temas: género, poder y dinero, un

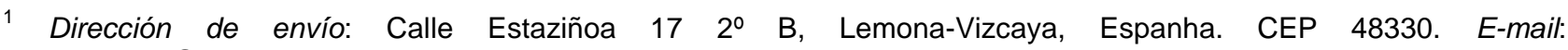
veraxavier13@gmail.com
} 
lenguaje de múltiples matices en las relaciones de pareja desde la perspectiva de la terapia familiar sistémica.

Las relaciones entre los sexos incluyen temas como sexualidad, ternura, etc., y al mismo tiempo también el trabajo, la profesión, la desigualdad, la política, la economía. Esta mezcla desequilibrada de todo, es lo que complica tanto las interrelaciones. (Beck, 2006).

\section{GÉNERO}

Las sociedades estructuran y construyen su cultura en torno a la diferencia sexual de los individuos que la conforman, atribuyéndoles características y significados a las acciones que desempeñan mujeres y hombres o se espera que desempeñen, según lo socialmente aprendido.

Pensando en cuestiones de género se puede entender también que la subordinación a la cual han estado sometidas las mujeres en diferentes periodos históricos, es producto de formas específicas de organización de estas sociedades donde lo femenino y lo masculino no son el resultado de una definición biológica sino la consecuencia de una desigualdad jerarquizada de las prácticas sociales, las funcionales y la ubicación que se tenga en la sociedad (Carranza, 2008).

Las relaciones entre las mujeres y el poder según (Prieur y Guillou (2007) es un tema indiscutiblemente complejo y multifacético. Por el lado de los hombres, estos también quieren mostrar sentimientos y debilidades. Sea cual fueren las particularidades presentadas en su matrimonio, hombres y mujeres son afectados por el sistema patriarcal que otorga privilegio a los hombres en detrimento de las mujeres.

\section{PAREJA Y DINERO}

El dinero juega indudablemente, un papel fundamental en las relaciones de pareja, aunque esté oficialmente prohibido hablar de este tema, ya que el amor y el dinero no se pueden mezclar, según dice el sentido común. Sin embargo, este tema está muy presente, en nuestra cultura el dinero aparece claramente sexuado y asociado a la potencia y a la virilidad, convertido en indicador de identidad sexual masculina (Coria, 2005).

Reflexionar sobre los diferentes modos de compartir el dinero en la pareja invita, a recordar que ambos cónyuges están previamente inscritos en una historia colectiva que se halla en continuo movimiento, una historia cuyos efectos todavía no se han medido del todo. No es posible entender las dificultades que los cónyuges tienen que afrontar en este tema, sin estudiar los profundos cambios experimentados por la célula familiar en las últimas décadas. En la actualidad en las relaciones aparecen las diferencias de culturas familiares, de ideas, de preconceptos y visiones de mundo (Fraenkel, 2003). Nos encontramos aquí, en un territorio aún por cartografiar.

\section{MÉTODO}

La metodología utilizada en nuestra investigación es un estudio de caso. Este es un método flexible de investigación que se ha utilizado para una gran cantidad de propósitos, que abarcan el desarrollo de la teoría de la evaluación y la educación (McLeod, 2010).

En la supervisión y la psicoterapia, el concepto de un "caso" se refiere a algún tipo de episodio de tratamiento en el que una persona (o grupo familiar) recibe ayuda de un terapeuta (Kasper, Hill y Kivlighan, 2008). La investigación en terapia se preocupa, por tanto, no sólo por lo que sucede en la vida del cliente o paciente, sino también por la aplicación de los conocimientos profesionales en la práctica del psicólogo (Lent, Hoffman, Hill, Treistman, Mount y Singley, 2006).

\section{OBJETIVO}

El objetivo general de este estudio es conocer la doble perspectiva de los litigantes durante el período previo a la separación judicial desde la interpretación de la estructura del texto obtenido a través de la transcripción de las entrevistas, que viabilizará aclarar nuevas posibilidades de actuación profesional ante una situación de toma de decisión.

\section{PROCEDIMIENTO}

Esta intervención consta de cinco encuentros, los participantes firmaran el Término de Consentimiento Informado, sus nombres son ficticios y tiene la aprobación del comité de ética.

\section{PARTICIPANTES}


La pareja Pablo (68 años de edad) y María (45 años de edad, nombres ficticios) viven en pareja desde hace 23 años, no están casados oficialmente, pero su unión es estable y tienen dos hijos, de 21 años y 19 años. Pablo tiene una furgoneta de reparto y no terminó los estudios en el instituto. Este es su segundo matrimonio, del primero tiene dos hijas. María, su esposa, no tiene trabajo. tampoco terminó los estudios primarios. Cuando tenía 17 años murió su madre y su padre les abandonó, son 6 hermanas, siendo ella la tercera.

\section{MOTIVO DE LA INTERVENCIÓN}

La pareja (Pablo y María) fue enviada por el Defensor público para que realizaran una intervención porque Pablo no aceptaba la separación. Pablo llora mucho cuando habla de separación. María dice que no quiere continuar más con esta relación, que él no la respeta, no reconoce lo que ella le ha ayudado, manifiesta que ella tiene que ser como él quiere que sea. Ella comenta que incluso pensó en quitarse la vida. Añade que participa de la intervención, pero que es la última oportunidad que le da a Pablo.

\section{Primera entrevista}

En la entrevista, María empieza diciendo que él sólo se fija en el $1 \%$ de las cosas, que son las que están mal y que siempre pone mala cara por ello. Ella dice que le quiere mucho, pero el sentimiento es distinto de lo que era al principio de la relación. Que perdió el gusto de hacer las cosas que quería, que se siente deprimida. Están de acuerdo cuando narran que son una pareja que ha trabajado mucho y que por esto tienen casa y su sustento. Pablo dice que sí, que es verdad, que siempre pone mala cara para todo. Dice que su error fue haberse ligado mucho al trabajo y que si no fuera por ella estaría sin dinero. María expone que su palabra nunca tenía valor, que hablaba y que no era escuchada. Cuenta que él también le prohibía ir a la iglesia. Ella dice que solamente vuelve a casa si es respetada como madre y como mujer.

En este momento se pone de manifiesto la extrema necesidad por parte de Pablo de que María vuelva a casa hablando solamente de los aspectos positivos de ella, con mucha exageración y estando de acuerdo con casi todo lo que ella decía.
P - “...Yo la amo locamente, ella para mí lo es todo. Tú eres mi madre, hoy tu eres mi madre. Entonces mi idea es que ahora sea ella la jefa de la casa".

En los varones, uno de los mecanismos discursivos más utilizados como forma de situar a la mujer en una posición relegada o dominada es la tendencia masculina a no atribuir en su discurso el papel de agente de las acciones a las mujeres, sino de objeto de las acciones masculinas. Este es un discurso que lo consideran propio de los varones para quienes el habla es un medio para preservar su independencia y negociar su estatus dentro de la jerarquía (Goldner, 1988).

María - "Yo estoy con depresión. Mi depresión es profunda, he adelgazado mucho. Él me prohibía ir a la iglesia".

El valor desigual atribuido por la sociedad a la contribución de hombres y mujeres se repite en la relación de la pareja. Al sentirse incapaces de asumir una posición de fuerza y auto-estima, las mujeres desarrollan modelos de comunicación indirectos que se manifiestan en explosiones de rabia, lamentaciones y disimulos. Cuando estas conductas no son eficaces las mujeres tienden a tornarse sintomáticas (Goodrich, Rampage, Ellman, y Halstead, 1988)

\section{Segunda entrevista}

Hicimos un análisis de la primera entrevistas y utilizamos los puntos más conflictivos en la devolución. Para intervenir con esta pareja se han señalados los siguientes aspectos: La relación jerárquica queda muy evidente en esta pareja ya que al contestar hasta en sus sueños María le mira a él como su padre, asumiendo un rol de niña en la relación.

María - "Hasta en mis sueños yo nunca soñé que él fuera mi marido, mi novio. En mi sueño él parece ser mi padre. Yo tengo miedo de él. Yo quería tanto una familia que yo idealice. Yo quería hacer de todo por tenerla."

Pablo ha tenido una rígida educación, nos habló mucho de su padre, diciendo que para él todo era pecado. Se presenta, todavía, muy obediente a los mandamientos del padre. En algunas conversaciones nos quedó esta duda, de que él podría ser su dueño, pero esto no lo teníamos muy claro, es una suposición. Este es un tema que no entendíamos muy bien en este momento.

Un tópico que aparece en la segunda entrevista es la cuestión de la separación de Pablo. 
Él nunca ha querido aproximar las dos familias. Cuenta María que las "cosas buenas" estaban en la "primera familia". María entonces comenta que se siente en segundo plano.

Pablo es un hombre dividido porque "tiene" que estar con las dos familias y no puede estar entero con ninguna, entendimos esto como un sufrimiento de Pablo. El primer aspecto derivado de las cuestiones familiares y culturales adquiridas en su rígida educación. El segundo, derivado de la culpa por la primera separación (Visher, Visher y Pasley, 2003).

\section{Tercera entrevista}

En este encuentro empiezan hablando de temas familiares. Opinan sobre el tema de la obediencia a los padres:

María: "Él me quitaba la autoridad delante de los niños. Hoy ellos son mayores, pero no me hacen caso...

Pablo: "Es falta de respeto, falta de autoridad. Ha llegado a un punto en el que yo no tengo autoridad. María no tiene autoridad. Hoy es mi hijo menor quien manda más que yo.

Pablo viene muy enfadado con María a este encuentro de la tercera entrevista. Entonces aparece una situación muy compleja. Pablo, por su enfado, nos cuenta que cuando conoció a María, ésta era trabajadora del sexo, y que él la sacó de ahí. Aquí aparece claramente porqué Pablo se siente "dueño de María" y que ella le debe estar agradecida toda la vida por haber hecho esto.

La prostitución es un ejemplo de cómo las personas transformadas en objeto son adquiridas a cambio de dinero (Coria, 2006). Por este aspecto de quién vende a alguien que se compra, el ejercicio de la prostitución se mantiene, estando los dos implicados. Esto nos esclarece la sospecha de que Pablo se siente dueño de María, como habíamos señalado anteriormente. En esta entrevista, Pablo llora mucho y María se queda más callada, no polemizando sobre este tema tan delicado.

En esta entrevista es donde queda demostrado que Pablo ejerce el poder activamente, no escucha las demandas de su mujer, afirma su autoridad, toma decisiones favorables a sus intereses personales y María acaba tolerando esas decisiones aunque no las considere adecuadas. En las relaciones con tanta proximidad es donde se manifiestan aspectos como el estrés, la ira y agresión verbal (Bodenmann, Meuwly, Bradbury, Gmelch y Ledermann, 2010).
Este encuentro nos posibilitó comprenderla mejor. También intentamos enseñar a Pablo que las relaciones son construidas y que, independiente del pasado de María, habían construido una familia y un trabajo digno juntos. Esto era lo importante para la pareja. La escucha activa comprende, escuchar el contenido, los hecho e ideas; escuchar la intención, ser consciente de los filtros emocionales que afectan a la comprensión del hablante $y$, escuchar al hablante sin hacer juicios y de forma comprensiva (Dugger, 2006).

Un marco político de referencia proporciona una perspectiva sobre el modo en la que se organiza la comunicación en un contexto social. Cuando el terapeuta hace preguntas con la intención de traer a la luz las reacciones de ciertas personas a las reacciones de otras personas, trabaja dentro de este marco (Keeney y Ross, 2007).

Al final de este encuentro, pedimos que trajesen una foto de la familia. Pensamos que así estimularíamos un diálogo, una demanda de María, menos conflictivo ya que, hablaríamos de los dos y de los hijos, utilizando como aspecto positivo la construcción de su familia (Daly, 2001).

\section{Cuarta entrevista}

En la cuarta entrevista María empieza contando que los dos discutieron en casa por los temas presentados en las entrevistas anteriores. Hablando ahora claramente de sus amarguras, nos cuenta María: "Cuando yo llego a casa él quiere que yo le abrace como sí no hubiera pasado nada."

La cuestión financiera es una forma de control utilizado por Pablo para mantener a María dependiente de él.

María: "Yo iba a la farmacia y cuando llegaba en casa tenía que darle el cambio. Después empecé a vender productos cosméticos para tener mis dineritos. Entonces él me ha dicho: ahora compras todo con tu dinero. Siempre así...Yo no trabajé fuera de casa, pero en las tareas domésticas yo siempre trabajaba. Ahora él dice que no tengo ningún derecho."

Le preguntamos de como se siente cuando no cuenta esto. María: "Mal, porque yo creo que sí él pudiera cobrarme hasta la comida, él me cobraba y después dice que no vive sin mí."

Se observa el doble mensaje que Pablo envía a María, pero para ella es difícil rebelarse. Cuando la dependencia de la mujer en relación al marido también es económica, las mujeres pueden sentirse avergonzadas y limitadas para desarrollar 
las habilidades necesarias para enfrentarse a la realidad extra familiar, una vez que en nuestra cultura las habilidades necesarias para la vida pública tienen mayor valor que la capacidad para administrar el hogar y la crianza de hijos (Addo y Sassler, 2010). Las habilidades que envuelven el cuidar y la empatía son frecuentemente desvaloradas 0 descalificadas (Blanton y Vandergriff-Avary, 2001).

Cuando nos enseña la foto que les pedimos, María esclarece uno de los motivos de no poder seguir adelante con la separación, que ya había intentado en otras veces que salió de casa. Tiene la ilusión de mantener una familia idealizada y "hace lo imposible", para que esa familia no desilusione.

Socializada para el cuidado, las mujeres están preparadas para ejercer los papeles destinados para ellas, desempeñando un rol central en la familia. Las mujeres deben moldearse a los intereses familiares, mantener la paz, mediar conflictos y ser tolerantes, incondicionales, altruistas y continentes (Bodenmann et al., 2010).

La separación para María está asociada a pérdida y muerte de la familia idealizada por ella. La familia que había perdido en su niñez con la muerte de su madre. Se queda en el matrimonio, siendo una situación que le trae más sufrimiento que realización. María presenta la dificultad de superar la pérdida de la madre, y de la familia idealizada, cuando nos cuenta un sueño, llorando mucho, que ha tenido con su madre, siendo uno de los momentos más emocionantes.

María: "Ella ha dicho ven que yo.... Tienes un sitio al que te voy a llevar, tus hijos se quedan, pero se quedarán bien no te preocupes... Mi madre me cogía por la mano y yo miraba y lloraba porque tenía que dejar a mis hijos." Trabajamos y reestructuramos empáticamente con María su sueño.

Pablo se encontraba muy deprimido y frustrado, según relato propio, porque se dio cuenta de que ahora María ya no cambiaría más de opinión en relación a la separación. Al final de este encuentro hablamos con Pablo para que él buscara un psiquiatra y pedimos a María que le ayudara. Le preguntamos si había alguno de sus hermanos y/o hermanas que también pudiera apoyarle.

\section{ENCUENTRO FINAL}

En la entrevista, ella dice que ahora está segura de que no quiere seguir la relación de esta forma. Nos comenta que le gustaría ser un pajarito, ir y venir cuando le diera la gana, porque había pasado mucho tiempo bajo el control de él. Él nos cuenta que ha ido al psiquiatra y que ahora empezaba a valorarse, que había estado humillándose mucho por ella. En algunos momentos se confundía y demostraba que la seguía queriendo, pero poco después demostraba su indignación porque ella le había fallado, según sus expectativas.

Ella nos dice que no quiere pelear con él, ya que tienen hijos y que si él aceptara la separación podría incluso cocinar, a veces, para ellos. Él dice que reza por ella, pero todavía demuestra mucha inconformidad con la separación. E están de acuerdo que sería bueno si todos de la familia pudiesen charlar, aunque piensan que, en estos momentos, se les pone difícil.

\section{INTERPRETACIÓN GENERAL DE LOS TEMAS Y CONCLUSIÓN}

Ésta es una pareja de hecho, de larga duración, pero con muchas implicaciones, como una separación anterior y secretos que fueron revelados a lo largo de la intervención. En relación a la situación financiera, tienen un nivel estable, pero una relación conflictiva con el reparto del dinero. Entre los conflictos elegimos el que nos parecía más urgente que era el abuso de poder por parte de Pablo conectado con la creencia, por parte de María, de la imposibilidad de salir de esta situación creyendo que la única salida posible era el suicidio, que ella misma verbalizó dos veces.

Pese a los cambios sociales que se han producido en las últimas décadas, el rol habitual de género y mujeres persiste la diferencia jerárquica entre hombres y mujeres persiste (Carranza, 2008). No obstante algunas mujeres ya empiezan a comprender y hacer un intento de salir de su situación de dependencia y sumisión. Cuando reestructuramos con María su relación proponiéndole una visión conceptual, basándonos en las diferencias de género, y emocional distinta a la que experimentaba la misma situación concreta, ella ha podido comprender y cambiar completamente su significado.

Se percibe que al final de la intervención María estaba segura de que no solamente quería salir de este matrimonio sino que además, ahora, se enfrenta a Pablo más que antes y podía aguantar "perder" su familia idealizada para quedarse con una familia real. En relación a Pablo, nuestro plan fue disminuir los perjuicios por la separación, le 
remitimos al psiquiatra para que se quedara respaldado a través de una evaluación y le propusimos un acercamiento a su familia de origen.

Se puede decir que esta pareja estableció una relación complementaria rígida (Watzlawick, Beavin, y Jackson, 1981), en donde sus roles son característicos de complementariedad, en la cual el hombre era el proveedor económico y administrador del dinero, mientras que la mujer cumplía las funciones "diarias" del género femenino, siendo su rol el de ama de casa. Sus disputas siempre pasaron por el hecho de que María quería romper con su rol y obtener más libertad y autonomía. Por lo tanto, se observa la disfuncionalidad del sistema conyugal. Pablo no acepta flexibilizar los roles y actúa para volver a la forma tradicional del funcionamiento de este sistema.

En los matrimonios más igualitarios, apuntaron Addo y Sassler (2010), que las parejas tienen la comunicación más positiva, la intimidad más profunda, el poder se distribuye más uniformemente y la autoestima es más alta debido a la distribución de derechos y deberes.

\section{CONCLUSIÓN}

Se pudo comprobar que en las negociaciones de la pareja se manifiestan las dificultades tanto las más explícitas como las encubiertas. Varones y mujeres se encuentran en una posición diferente a la hora de adoptar decisiones. La inferioridad social de las mujeres les impide negociar en igualdad de condiciones, volviéndose la tarea todavía más ardua para ellas, cuando quieren separarse.

La constitución de la pareja pasa por el prisma de la complejidad y de la singularidad del vivir humano. De esta manera se hace necesario que se preparen los profesionales, para intervenir, con una visión también volcada a los aspectos culturales, sociales, de género y económicos del ser humano.

\section{REFERÊNCIAS}

Addo, F.R., y Sassler, S. (2010). Financial arrangements and relationship quality in lowIncome. Family Relations, 59, 408-423.

Beck, U. (2006). La sociedad del riesgo. Hacia una nueva modernidad. Barcelona: Paidós.

Blanton, P.W., y Vandergriff-Avery, M. (2001). Marital therapy and marital power: Constructing narratives of sharing relational and positional power. Contemporary Family Therapy, 23(3), 295-308.

Bodenmann, G., Meuwly, N., Bradbury, T.N., Gmelch, S., y Ledermann, T. (2010). Stress, anger, and verbal aggression in intimate relationships: Moderating effects of individual and dyadic coping. Journal of Social and Personal Relationships, 27, 408-424.

Carranza, M. (2008). Therapeutic interventions to domestic violence with immigrant couples. Intimate partner and family abuse: A casebook of gender-inclusive therapy. New York: Springer Publishing Co.

Coria, C. (2005). El dinero en la pareja. Algunas desnudeces sobre el poder (2a ed.). Buenos Aires: Paidós.

Coria, C. (2006). El sexo oculto del dinero. Formas de la dependencia femenina (5a ed.). Buenos Aires: Paidós.

Daly, K.J. (2001). Deconstructing family time: from ideology to lived experience. Journal of Marriage and Family, 63, 283-294.

Dugger, J. (2006). Escucha eficaz: la clave de la comunicación. Madrid: Fundación Confemetal.

Fraenkel, P. (2003). Contemporary two-parent families. Navigating work on family challenges. En. F. Walsh (Ed.), Normal family processes (pp. 61-95). New York: Guilford Press.

Goldner, V. (1988). Generation and gender: normative and covert hierarchies. Family Process, 27, 17-31.

Goodrich, T.J., Rampage, C., Ellman, B., y Halstead, K. (1988). Feminist family therapy: A casebook. New York: Norton.

Kasper, L., Hill, C.E., y Kivlighan, D. (2008). Therapist immediacy in brief psychotherapy therapy: Case study I. Psychotherapy: Theory, Research, Practice, Training, 45(3), 281-297.

Keeney, B.P., y Ross, J.M. (2007). Construcción de terapias familiares sistémicas. Espíritu en la terapia (2a ed.). Buenos Aires: Amorrortu.

Lent, R. L., Hoffman, M. A., Hill, C. E., Treistman, D., Mount, M., y Singley, D. (2006). Clientspecific counselor self-efficacy in novice counselors: Relation to perceptions of session quality. Journal of Counseling Psychology, 53(4), 453-463.

McLeod, J. (2010). Case Study Research. In counseling and psychotherapy. London: Sage.

Prieur, B., y Guillou, S. (2008). Dinero y pareja. ¿Es posible amar sin hacer cuentas? Barcelona: Urano.

Visher, E., Visher, J. y Pasley, K. (2003). Remarriage families and stepparenting. En. F. Walsh (Ed.), Normal family processes (pp.153175). New York: Guilford Press.

Watzlawick, P., Beavin, J., y Jackson, D. (1981). Teoría de la Comunicación Humana. Barcelona: Herder. 
Recebido em 05/09/2012

Aceito em 25/02/2013

Vera Regina Xavier: doctora en Psicologia, Programa Salud y Familia, Universidad de Deusto, España, presidente de la Asociación Intercultural de Apoyo Emocional a la Diversidad Social, Bilbao, España. 